\title{
O TRABALHO DOCENTE E A SAÚDE DO PROFESSOR UNIVERSITÁRIO: UMA REVISÃO SISTEMÁTICA
}

\section{THE TEACHING WORK AND THE HEALTH OF THE UNIVERSITY TEACHER: A SYSTEMATIC REVIEW}

\author{
Gláucia Guimarães de Souza Neme \\ Mestre, Programa de Pós-graduação em Saúde Ambiental e Saúde do Trabalhador \\ Universidade Federal de Uberlândia \\ glauguimaraes@yahoo.com.br \\ Jean Ezequiel Limongi \\ Doutor, Programa de Pós-graduação em Saúde Ambiental e Saúde do Trabalhador \\ Universidade Federal de Uberlândia \\ jeanlimongi@gmail.com
}

\begin{abstract}
RESUMO
Objetivo: o presente estudo teve como objetivo realizar uma revisão sistemática para avaliar a relação entre condições organizacionais de trabalho e queixas e/ou agravos à saúde em docentes do ensino superior. Métodos: Foram realizadas buscas em cinco bases de dados por meio das palavras-chave "professores universitários", "riscos ocupacionais", "saúde do trabalhador" e Brasil. Foram incluídos artigos originais de pesquisa, dissertações de mestrado e teses de doutorado, publicados no período compreendido entre 2000 e 2016 no idioma inglês e português. Resultados: a revisão identificou 162 estudos que abordavam a temática, dos quais 22 atenderam plenamente aos critérios estabelecidos. $\mathrm{O}$ estudo evidenciou diversos fatores do trabalho interferindo no estado de saúde dos docentes, desencadeando agravos, com destaque para alterações da saúde mental, mas também apontou realização pessoal no desenvolvimento das atividades laborais e qualidade de vida satisfatória. Conclusão: o adoecimento entre professores universitários ocorre, principalmente, devido à exaustão ocasionada pelo modo de produção do trabalho, que impõe uma sobrecarga de atividades, deixando-os em situação de vulnerabilidade.
\end{abstract}

Palavras - chave: Professores universitários. riscos ocupacionais. saúde do trabalhador

\begin{abstract}
Objective: the present study aimed to conduct a systematic review to evaluate the relationship between organizational working conditions and health complaints and / or grievances in higher education teachers Methods: Five databases were searched through the keywords "university professors", "occupational risks", "worker's health" and Brazil. Were included original articles, master dissertations and doctoral theses, published between 2000 and 2016 in the English and Portuguese languages. Results: the review identified 162 studies that addressed the subject, of which 22 met the established criteria. The study evidenced several factors of the work interfering in the state of health of the teachers, triggering aggravations, with emphasis on changes in mental health, but also pointed personal fulfillment in the development of work activities and satisfactory quality of life Conclusion: the sickness among university professors occurs mainly due to the exhaustion caused by the way of production of the work, which imposes an overload of activities, leaving them in a situation of vulnerability.
\end{abstract}

Keywords: University professors. occupational risks. worker health.

Recebido em: 01/08/2019

Aceito para publicação em: 28/08/2019. 


\section{INTRODUÇÃO}

O estudo das condições de saúde e de trabalho de grupos ocupacionais permite caracterizar os processos laborais e descrever o perfil de adoecimento dos trabalhadores, avaliando possíveis associações entre ocupação e saúde (ARAÚJO et al., 2005).

A literatura sobre as condições de trabalho e saúde dos professores ainda é bastante limitada no Brasil, principalmente no âmbito universitário, quando comparada a outras atividades laborativas (ARBEX; SOUZA; MENDONÇA, 2013; LIMA; LIMA FILHO, 2009). Os estudos, em geral, destacam o processo de adoecimento nos contextos fabris, onde a relação entre trabalho e doença ocupacional é mais direta e os riscos à saúde mais evidentes (ARAÚJO et al., 2005). Obviamente, as condições gerais de saúde da classe docente envolve uma miríade de fatores que se interligam de forma complexa e que, em essência, são influenciados por fatores de âmbito social e particularidades da área como a Instituição em que trabalha (público/privada), localização (zona rural/urbana; periferias) e nível de ensino (básico, médio, superior). No ensino superior, a necessidade de atuação em atividades de ensino, pesquisa, extensão e gestão têm ocasionado grande desgaste da atividade laboral dos professores e, em decorrência deste processo, tem ocorrido um aumento do adoecimento nessa categoria profissional (AMORIM; FREITAS; GOMES, 2017; LEITE; NOGUEIRA, 2017; MARIN, 2010; REIS; CECÍLIO, 2014).

As pesquisas existentes sobre a saúde de professores, em geral, apontam principalmente as doenças ocupacionais clássicas como os distúrbios da voz e as doenças musculoesqueléticas (RIBEIRO, 2015; ALVES, 2011; LEMOS, 2005). São frequentes também o estresse ocupacional, associado a sentimentos de hostilidade, tensão, ansiedade, frustração e depressão desencadeados por estressores localizados no ambiente de trabalho (ARAÚJO; MARTINS, 2015; REIS, 2006; ANDRADE; CARDOSO, 2012). Fatores como o fraco apoio social e o ambiente agitado em função da indisciplina dos alunos contribuem sobremaneira neste processo (ASSUNÇÃO; ABREU, 2019). Santos e Neves (2017) destacam a negligência das instituições de pesquisa e de saúde pública em relação ao adoecimento da classe docente e chamam atenção para a necessidade de ações e políticas para mudar esta realidade.

O objetivo do presente estudo foi realizar uma revisão sistemática para avaliar a relação entre condições organizacionais de trabalho e as queixas e/ou agravos à saúde em docentes do ensino superior.

\section{MATERIAL E MÉTODOS}

Foi realizada uma revisão sistemática de estudos que abordaram, por meio de inquérito epidemiológico, a associação entre condições da organização de trabalho e saúde do professor universitário.

Foram incluídos nesta revisão sistemática artigos originais de pesquisa, dissertações de mestrado e teses de doutorado, conforme recomendado por PEREIRA \& GALVÃO (2014), publicados no período compreendido entre 2000 e 2016, nos idiomas português e inglês, resultantes de investigações realizadas no Brasil e que apresentaram clareza dos seguintes critérios de inclusão: (i) informações sobre a população estudada - professores do ensino superior da rede pública ou privada; (ii) dados sobre as condições gerais do trabalho docente no ensino superior; (iii) metodologia de levantamento de dados por meio de inquéritos epidemiológicos (busca ativa), com análise descritiva ou inferencial; (iv) caracterização dos desfechos na saúde dos professores universitários.

Quanto ao processo operacional da pesquisa, as fases de leitura e avaliação dos títulos, resumos e textos integrais, de extração de dados e de elaboração da síntese foram conduzidas por dois pesquisadores.

As publicações foram pesquisadas nas bases de dados CAPES/MEC, Google Scholar, Medline/Pubmed, Scielo e Lilacs/Bireme. Os descritores utilizados, combinados entre si, em busca integrada nos campos título, resumo e assunto foram as seguintes: "professores universitários" OR "university professors" AND "riscos ocupacionais" OR "occupational risks" AND "saúde do trabalhador" OR "worker health" AND Brasil OR Brazil. Foi utilizada a mesma estratégia de busca em todas as bases de dados. 
Os dados encontrados foram tabulados, em planilha eletrônica, descrevendo-se os autores, natureza do trabalho, ano de publicação, desenho do estudo, número de participantes da pesquisa, distribuição por sexo, intervalo de faixa etária e desfecho do estudo.

A leitura dos trabalhos, coleta dos dados e extração das informações foram realizadas de modo independente entre os dois pesquisadores. Divergências foram identificadas, discutidas e resolvidas entre os pesquisadores, sem necessidade de consultar um terceiro revisor.

Foi utilizada a ferramenta PRISMA (Preferred Reporting Items For Systematic Reviews And MetaAnalyses) para orientar a redação da presente revisão sistemática.

\section{RESULTADOS E DISCUSSÃO}

O processo de busca e seleção dos estudos que compõem a presente revisão está representado na Figura 1.

Figura 1 - Representação esquemática do processo de análise de artigos para revisão sistemática.
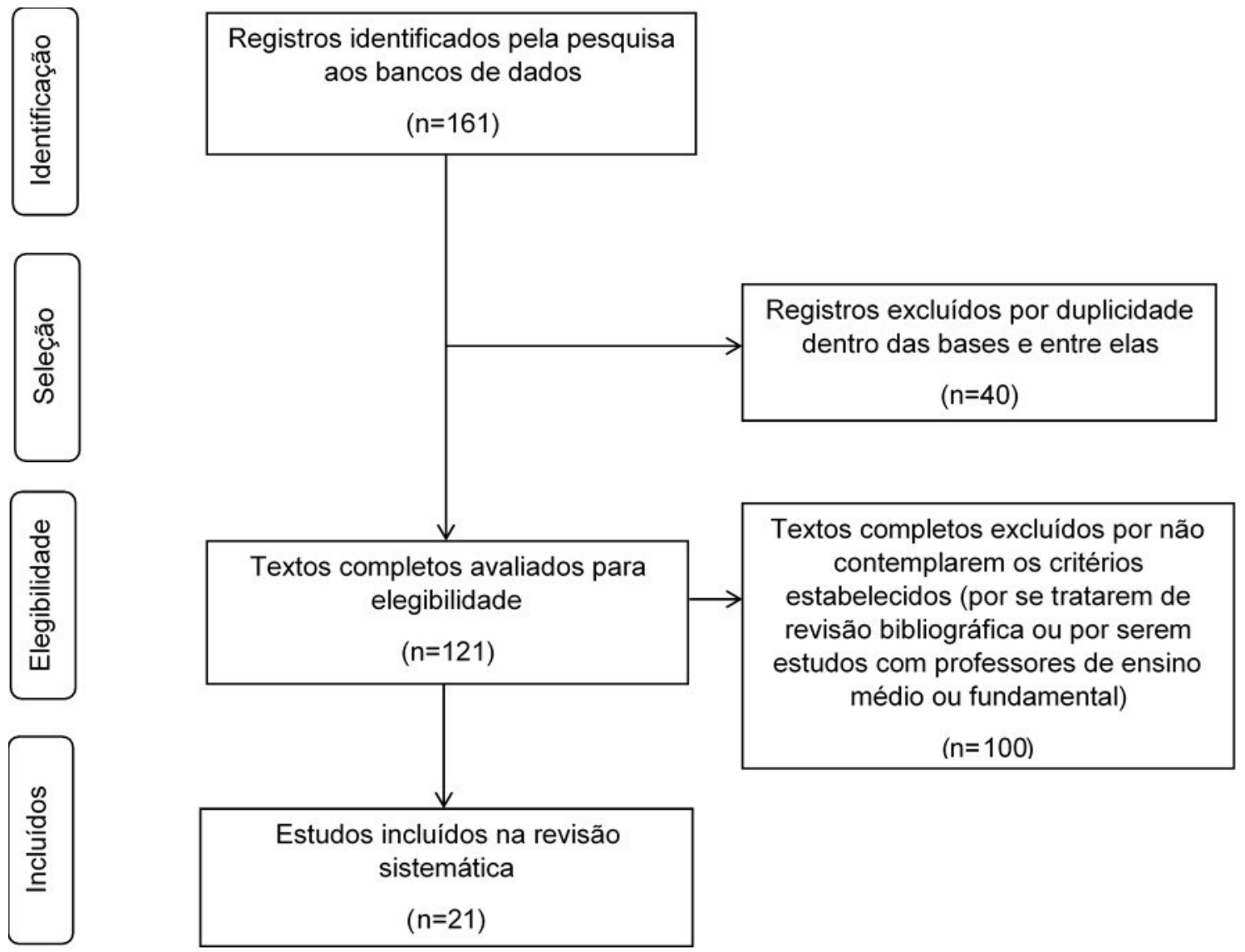

A consulta às bases de dados eletrônicas demonstrou um escasso número de publicações a respeito do estado de saúde do docente universitário. Grande parte da literatura identificada com os descritores pesquisados pertencia a "literatura cinza", a qual é representada por dissertações de mestrado e teses de doutorado. Dentre os 22 trabalhos selecionados, oito eram artigos, dez eram dissertações e quatro eram teses (PEREIRA; GALVÃO, 2014). 
A caracterização da fonte de dados, do desenho do estudo, da amostra estudada e desfecho da associação entre condições de trabalho e saúde dos professores universitários são apresentadas na Tabela 1. (Anexo 1).

Em relação aos métodos e aos instrumentos utilizados nos trabalhos selecionados, foram aplicados questionários $(14 ; 63,6 \% \%)$, entrevistas $(4 ; 18,2 \%)$, um estudo sobre capacidade vocal $(1 ; 4,5 \%)$ e levantamentos de dados secundários nos sistemas SIASS (Subsistema Integrado de Assistência à Saúde do Servidor) e SIAPE (Sistema Integrado de Administração de Recursos Humanos), ambos de servidores federais $(3 ; 13,6 \%)$. Os estudos foram na sua maioria de natureza quantitativa e de corte transversal, primordialmente $(90,5 \%)$.

O número de participantes nos estudos variou de 7 a 2218 e a faixa etária foi de 20 a 75 anos de idade. Houve um maior percentual de profissionais do sexo feminino nas amostras pesquisadas $(60 \%)$.

De acordo com os resultados apresentados, o ambiente organizacional de trabalho interfere com o estado de saúde dos docentes, devido principalmente aos riscos psicossociais presentes no ambiente acadêmico.

Queixas de dores osteomusculares e distúrbios da voz foram relacionadas a condições ergonômicas do trabalho, ressaltando-se a postura ortostática na jornada de trabalho e necessidade de carregar peso, como o material didático, por exemplo (LIMA; LIMA FILHO, 2005; SERVILHA; ARBACH, 2011; AUAD, 2007, ALVES, 2011, LIMA; SILVA, 2014, FONTANA; RIBEIRO, 2010). As doenças osteomusculares não foram relacionadas à idade e altura (SANCHEZ, 2013). Em relação ao tempo da jornada de trabalho, houve controvérsia, entre os resultados dos trabalhos identificados. Em SANCHEZ et al (2013) a incidência de dores musculoesqueléticas nos docentes foi independente do tempo e jornada de trabalho, já em LIMA e LIMA FILHO (2014), esta associação foi constatada. Provavelmente, isto se deve ao fato de diferenças no ambiente de trabalho onde os estudos foram realizados.

Em relação às condições ambientais, o destaque como predisponente de agravos à saúde foi o ruído (RIBEIRO, 2015; ALBUQUERQUE, 2013) que pode ser proveniente da própria sala de aula ou de outras fontes externas. Em trabalho que comparou docentes de instituições de ensino superior (IES) público e privada, constatou-se que a exposição à poeira e produtos químicos é mais comum em IES públicas. Os autores neste estudo inferem que estes resultados se devem às melhores condições de conservação e limpeza dos espaços e uso de equipamentos de proteção individual nas IES privadas e talvez pela maior frequência de cursos com manipulação de produtos químicos nas IES públicas (SOUSA, 2013).

As pesquisas apontaram os transtornos mentais e comportamentais, juntamente com as doenças osteomusculares e do tecido conjuntivo, como importante causa de afastamentos das atividades laborais por parte dos docentes (COSTA et al., 2013) tanto em IES públicas quanto privadas, com um crescimento acentuado dos distúrbios psiquiátricos, como a síndrome de Burnout ou esgotamento profissional, além de alcoolismo, dependência química, transtornos depressivos e ansiosos, assédio moral e, em casos extremos, suicídio, como fatores causadores do absenteísmo no trabalho (COSTA, 2016; MARTINS, 2007) No estudo de MARTINS (2007) foi verificada maior predisposição ao desenvolvimento da síndrome de Burnout entre docentes mais jovens e naqueles com maior formação educacional.

O acúmulo de atribuições e responsabilidades foi identificado como causador do aumento de morbidades e absenteísmo uma vez que o trabalho docente não se limita somente às atividades em sala de aula, tampouco ao atendimento ao aluno, pelo contrário, abrange questões de ordem administrativas, como gestão, além de desenvolvimento de projetos de pesquisa e extensão, sendo na maioria das instituições avaliados segundo o número de publicações de artigos científicos (RIBEIRO, 2016).

Também foi apontado que a desproporcionalidade entre o salário dos professores e suas responsabilidades repercute em sua saúde física e mental, havendo grande sobrecarga de trabalho dentro e fora do ambiente acadêmico, cobranças, dificuldades de relacionamento com a chefia e com os alunos, como fatores de estresse, prejudicando sua saúde (RIBEIRO, 2015; DALAGASPERINA; MONTEIRO, 2016). Em trabalho que comparou docentes de IES públicas e privadas, verificou-se que 
ambos estão expostos a fatores desconfortáveis para o desenvolvimento de suas atividades, sendo o acúmulo de atividades uma característica dos docentes de IES privadas (SOUSA, 2013).

Contudo vale ressaltar que, ainda que algumas situações do trabalho estejam relacionadas à ocorrência de agravos à saúde, também é notável que o desenvolvimento da atividade em sala de aula proporciona grande satisfação pessoal, mesmo que os docentes apresentem sintomas da síndrome de Burnout em períodos de maior demanda (PEREIRA, 2006). Em trabalho que comparou escores de autoestima de docentes de IES pública e privada, foi apontada maior média entre professores da instituição pública, porém, em outro estudo que avaliou qualidade de vida em docentes de uma faculdade privada, os índices foram elevado (TERRA; MARZIALE; ROBAZZI, 2013; LOURENÇO, 2009).

No estudo de OLIVEIRA (2001), os fatores identificados como influenciadores do bem-estar docente foram, em ordem crescente de importância: respeitabilidade, família, amigos, autoestima, saúde, satisfação no trabalho, situação financeira, lazer, participação em grupos e religiosidade. Em estudo realizado entre docentes de enfermagem, no sul do país, observou-se correlação positiva entre resiliência e qualidade de vida (MIGUEL, 2012).

Do ponto de vista metodológico, ainda que o número de publicações no formato de artigos seja limitado, o presente estudo, baseado também em dissertações e teses, permitiu visualizar o panorama geral do modo de produção do trabalho docente, as condições de trabalho às quais estão sujeitos e a interferência desta situação no seu estado de saúde.

\section{CONCLUSÃO}

Os resultados encontrados na revisão sistemática permitem verificar uma tendência ao adoecimento entre professores universitários, devido à exaustão ocasionada pelo modo de produção do trabalho, que impõe uma sobrecarga de atividades, deixando-os em situação de vulnerabilidade.

Diante do exposto, entende-se que deve ser revista a forma de trabalho do docente universitário, cabendo às instituições de ensino, públicas e privadas, fornecer melhores condições físicas de trabalho, adotando estratégias para proporcionar condições ergonômicas satisfatórias e redução dos riscos ocupacionais no ambiente de trabalho desses profissionais, minimizando os efeitos dos fatores geradores de doenças osteoarticulares, distúrbios da voz e estresse.

Devido à importância do cuidado com a saúde do trabalhador, a viabilização de práticas para a promoção da saúde, como ginástica laboral, conforto ambiental, relação de trabalho adequada entre colegas e chefia, bem como a prevenção da saúde de docentes, com a realização de exames periódicos de rotina, devem ser estimuladas, de forma a manter o estado geral de saúde físico, psíquico e social, com a preservação da autoestima e consequente manutenção da qualidade de vida do profissional.

\section{REFERÊNCIAS BIBLIOGRÁFICAS}

ALBUQUERQUE, A. L. Relação entre condições de trabalho e saúde vocal de professores no ensino superior [Dissertação] Vitória de Santo Antão (PE): Universidade Federal de Pernambuco. 2013.

ALVES, L. A. Uso prolongado da voz em professoras universitárias: uma questão de saúde do trabalhador [Tese]. Ribeirão Preto (SP): Universidade de São Paulo; 2011.

AMORIM, S. T.; FERREIRA, T. A.; GOMES, N. M. L. Influência da Fadiga Ocupacional na Capacidade para o Trabalho de Professores Universitários. Ciencia \& Trabajo, Santiago, v. 19, n. 59,p. 86-90, 2017. https://doi.org/10.4067/S0718-24492017000200086

ANDRADE, P.; CARDOSO, T. Prazer e dor na docência: revisão bibliográfica sobre a síndrome de Burnout. Saúde \& Sociedade, São Paulo, v. 21, n.1, p. 129-40, 2012. https://doi.org/10.1590/S0104$\underline{12902012000100013}$ 
ARAUJO, M. M. S.; MARTINS, F. C. S. Intensificação do trabalho e percepção da saúde em docentes de uma Universidade pública Brasileira. Ciencia \& Trabajo, Santiago, v. 17, n. 54 ,p. 212-218, 2015. https://doi.org/10.4067/S0718-24492015000300010

ARAÚJO, T. M et al. Mal-estar docente: avaliação de condições de trabalho e saúde em uma instituição de ensino superior. Revista Baiana Saúde Pública, v. 29, n. 1, p. 6-21, 2005.

ARBEX, A. S.; SOUZA, K. R.; MENDONÇA, A. L. O. Trabalho docente, readaptação e saúde: a experiência dos professores de uma universidade pública. Physis Revista de Saúde Coletiva, Rio de Janeiro, v. 22, n. 1, p. 263-284, 2013. https://doi.org/10.1590/S0103-73312013000100015

ASSUNÇÃO, A. A.; ABREU, M. N. S. Pressão laboral, saúde e condições de trabalho dos professores da Educação Básica no Brasil. Cadernos de Saúde Pública, v. 35, Sup 1: e00169517, 2019. https://doi.org/10.1590/0102-311x00169517

AUAD, A. R. B. Interferência de fatores ambientais e emocionais na voz de docentes universitários. [Dissertação]. Goiânia (GO): Pontifícia Universidade Católica de Goiás; 2007.

COSTA, D. L. Análise da relação entre saúde mental e trabalho de docentes universitários [Dissertação]. Natal (RN): Universidade Federal do Rio Grande do Norte; 2016.

COSTA, L. S. T. et al. Prevalência da Síndrome de Burnout em uma amostra de professores universitários brasileiros. Psicologia Reflexão Critica, Porto Alegre, v.26, n. 4, p. 636-642, 2013. https://doi.org/10.1590/S0102-79722013000400003

DALAGASPERINA, P.; MONTEIRO, J. K. Estresse e docência: um estudo no ensino superior privado. Revista Subjetividades, São Paulo, v. 16, n. 1, p. 37-51, 2016. https://doi.org/10.5020/23590777.16.1.37-51

FONTANA ,R. T.; PINHEIRO, D. A. Condições de saúde auto referidas de professores de uma universidade regional. Revista Gaúcha de Enfermagem, Porto Alegre, v. 31, n. 2, p. 270-276, 2010. https://doi.org/10.1590/S1983-14472010000200010

LEMOS, J. C. Cargas psíquicas no trabalho e processos de saúde em professores universitários. [Tese]. Florianópolis (SC): Universidade Federal de Santa Catarina; 2005.

LEITE, A. F.; NOGUEIRA, J. A. D. Fatores condicionantes de saúde relacionados ao trabalho de professores universitários da área da saúde: uma revisão integrativa. Revista Brasileira de Saúde Ocupacional, São Paulo, v. 42, e6, 2017. https://doi.org/10.1590/2317-6369000010116

LIMA, J. P.; SILVA, T. F. Análise da sintomatologia de distúrbios osteomusculares em docentes da Universidade de Pernambuco - Campus Petrolina. Revista Dor, São Paulo, v. 15, n. 4, p. 276-280 2014.

LIMA, M. F. E. M.; LIMA FILHO, D. O. Condições de trabalho e saúde do/a professor/a universitário/a. Revista Ciências \& Cognição, Rio de Janeiro, v. 14, n. 3, p. 62-82. 2009.

LOURENÇO, N. C. F. Qualidade de vida no trabalho de docentes de uma faculdade privada no município de São Paulo [Dissertação]. Guarulhos (SP): Universidade de Guarulhos; 2009.

MARIN, A. J. Precarização do trabalho docente. In: Oliveira DA; DMC; Vieira LMF. Dicionário: trabalho, profissão e condição docente. Belo Horizonte: UFMG/Faculdade de Educação, 2010.

MARTINS, R. M. O indicativo da incidência Burnout em professores do ensino superior: como prevenir ou remediar? [Dissertação]. Itajaí (MG): Universidade do Vale do Itajaí; 2007.

MIGUEL, M. E. G. B. Resiliência e qualidade de vida de docentes de enfermagem [Tese] Ribeirão Preto (SP): Universidade de São Paulo; 2012.

OLIVEIRA, C. R. B. Bem-estar dos docentes de uma instituição privada de ensino superior [Tese] Florianópolis (SC): Universidade Federal de Santa Catarina; 2001.

PEREIRA, M. G.; GALVÃO, T. F. Etapas de busca e seleção de artigos em revisões sistemáticas da literatura. Epidemiologia \& Serviços de Saúde, Brasília, v. 23, n. 2, p. 369-71, 2014. https://doi.org/10.5123/S1679-49742014000200019 
PEREIRA, O. A. V. Qualidade de vida no trabalho de docentes universitários de uma instituição pública e outra privada do leste de Minas Gerais [Dissertação]. Caratinga (MG): Centro Universitário de Caratinga; 2006.

REIS, B. M.; CECÍLIO, S. Precarização, trabalho docente intensificado e saúde de professores universitários. Trabalho \& Educação, Rio de Janeiro, v. 23, n. 2, p. 109-128, 2014. https://doi.org/10.1590/S0101-73302006000100011

REIS, E. J. F. B et al. Docência e exaustão emocional. Educação \& Sociedade, São Paulo, v.27, n. 94, p. 229-253, 2006.

RIBEIRO, A. Z. C. R. Perfil de morbidade do docente universitário de uma instituição federal de ensino superior [Dissertação]. Alfenas (MG): Universidade Federal de Alfenas; 2016.

RIBEIRO, L. H. Ambiente sonoro e a qualidade de vida vocal de professores universitários [Dissertação]. Curitiba (PR): Universidade de Tuiuti do Paraná; 2015.

SANCHEZ, $\mathrm{H}$. M et al. Incidência de dor musculoesquelética em docentes do ensino superior. Revista Brasileira de Medicina do Trabalho, São Paulo, v. 11, n. 2, p. 66-75, 2013.

SANTOS, F. A. L.; NEVES, I. R. Saúde do trabalhador em educação: a gestão da saúde de professores de escolas públicas brasileiras. Saúde \& Sociedade, São Paulo, v.26, n.3, p.786-797, 2017. https://doi.org/10.1590/s0104-12902017167259

SERVILHA, E. A. M,; ARBACH, M. P. Queixas de saúde em professores universitários e sua relação com fatores de risco presentes na organização do trabalho. Distúrbios da Comunicação, São Paulo, v. 23, n. 2, p. 181-191, 2011.

SOUSA, A. M. O perfil do adoecimento docente na universidade de Brasília de 2006 a 2011. [Dissertação]. Brasília (DF): Universidade de Brasília; 2013.

TERRA, F. S.; MARZIALE, M. H. P.; ROBAZZI, M. L. C. C Avaliação da autoestima em docentes de enfermagem de universidade pública e privada. Revista Latino-Americana de Enfermagem, São Paulo, v. 21, 2013. 
Anexo 1: tabela 1 - Caracterização da fonte de dados, do desenho do estudo, da amostra estudada e desfecho da associação entre condições de trabalho e saúde dos professores universitários na literatura selecionada.

\begin{tabular}{|c|c|c|c|c|c|c|}
\hline $\begin{array}{c}\text { Natureza do trabalho e } \\
\text { autores }\end{array}$ & $\begin{array}{c}\text { Ano de } \\
\text { publicação }\end{array}$ & $\begin{array}{l}\text { Desenho do } \\
\text { estudo }\end{array}$ & $\begin{array}{c}\text { Número de } \\
\text { participantes }\end{array}$ & $\begin{array}{l}\text { Faixa etária } \\
\text { (anos) }\end{array}$ & $\begin{array}{l}\text { Distribuição por } \\
\text { sexo }\end{array}$ & Desfecho do trabalho \\
\hline $\begin{array}{l}\text { Tese de doutorado } \\
\text { OLIVEIRA CRB }\end{array}$ & 2001 & Transversal & 60 & Não relatado & $\begin{array}{l}31,8 \% \mathrm{~F} \\
68,2 \% \mathrm{M}\end{array}$ & $\begin{array}{l}\text { Os fatores que mais influenciaram no bem-estar docente de } \\
\text { uma universidade privada, em ordem crescente de importância, } \\
\text { foram: respeitabilidade, família, amigos, autoestima, saúde, } \\
\text { satisfação no trabalho, situação financeira, lazer, participação } \\
\text { em grupos e religiosidade. }\end{array}$ \\
\hline $\begin{array}{l}\text { Tese de doutorado } \\
\text { LEMOS JC }\end{array}$ & 2005 & Transversal & 86 & $21-64$ anos & $\begin{array}{l}64 \% \mathrm{~F} \\
36 \% \mathrm{M}\end{array}$ & $\begin{array}{l}\text { A dor e o desconforto referidos pelos professores estão } \\
\text { relacionados à precariedade das condições de trabalho. Em } \\
\text { relação às condições físicas, destacam-se o ruído, a exigência } \\
\text { de posturas desequilibradas e da voz. No que se refere às } \\
\text { condições organizacionais, a desproporcionalidade entre o } \\
\text { salário do professor e suas responsabilidades repercute na sua } \\
\text { saúde física e mental. }\end{array}$ \\
\hline $\begin{array}{c}\text { Dissertação de mestrado } \\
\text { PEREIRA OAV }\end{array}$ & 2006 & Transversal & 36 & Não relatado & $\begin{array}{l}22,8 \% \mathrm{~F} \\
78,2 \% \mathrm{M} \\
\\
41,7 \% \mathrm{~F} \\
58,3 \% \mathrm{M}\end{array}$ & $\begin{array}{l}\text { Estudo de qualidade de vida no trabalho em IES pública e } \\
\text { privada, ambos grupos demonstram satisfação, porém com } \\
\text { sintomas de Síndrome de Burnout em períodos de maior } \\
\text { demanda. }\end{array}$ \\
\hline $\begin{array}{c}\text { Dissertação de mestrado } \\
\text { AUAD ARG }\end{array}$ & 2007 & Transversal & 150 & $25-65$ anos & $\begin{array}{l}65 \% \mathrm{~F} \\
35 \% \mathrm{M}\end{array}$ & $\begin{array}{l}\text { A precariedade das condições de trabalho, as falhas na } \\
\text { infraestrutura das universidades e as estratégias de } \\
\text { enfrentamento usados pelos docentes, constituem fatores } \\
\text { determinantes para o desencadeamento de alterações vocais. }\end{array}$ \\
\hline $\begin{array}{c}\text { Dissertação de mestrado } \\
\text { MARTINS RM }\end{array}$ & 2007 & $\begin{array}{c}\text { Transversal } \\
\text { (quanti-qualitativo) }\end{array}$ & 50 & $\begin{array}{l}25-60 \text { anos } \\
\text { ou mais }\end{array}$ & $\begin{array}{l}40 \% \mathrm{~F} \\
60 \% \mathrm{M}\end{array}$ & $\begin{array}{l}\text { O estudo aponta maior predisposição ao desenvolvimento da } \\
\text { síndrome de Burnout entre docentes mais jovens e naqueles } \\
\text { com nível educacional mais elevado. Foram identificados } \\
\text { fatores indicativos de incidência positiva para a síndrome, em } \\
\text { uma faculdade privada, de acordo com o instrumento de } \\
\text { investigação, denominado Diagnóstico Integrado de Trabalho. }\end{array}$ \\
\hline $\begin{array}{l}\text { Dissertação de mestrado } \\
\text { LOURENÇO NCF }\end{array}$ & 2009 & Transversal & 28 & $26-60$ anos & $\begin{array}{l}46,43 \% \mathrm{~F} \\
53,57 \% \mathrm{M}\end{array}$ & $\begin{array}{l}\text { O estudo mostrou um alto índice de satisfação entre docentes } \\
\text { de uma universidade privada, com médias elevadas, variando } \\
\text { entre } 71,4 \text { e } 94,0 \% \text { de satisfação com a qualidade de vida no } \\
\text { trabalho, ainda que } 71 \% \text { tenha outro emprego. }\end{array}$ \\
\hline $\begin{array}{l}\text { Artigo } \\
\text { FONTANA RT, PINHEIRO } \\
\text { DA }\end{array}$ & 2010 & Transversal & 34 & $\begin{array}{l}\text { Idade média de } \\
\quad 40,6 \text { anos }\end{array}$ & $\begin{array}{l}62 \% \mathrm{~F} \\
38 \% \mathrm{M}\end{array}$ & $\begin{array}{l}\text { Lombalgia e cansaço foram queixas prevalentes. Posição } \\
\text { ortostática, carregar material didático e trabalhar sob condições } \\
\text { ergonômicas inadequadas podem justificar tais agravos. }\end{array}$ \\
\hline DOI:http://dx.doi.org/10.1 & 3/Hygeia1 & 61 & & Hygeia & v.16 & p.1 - 10, \\
\hline
\end{tabular}




\begin{tabular}{|c|c|c|c|c|c|c|}
\hline $\begin{array}{l}\text { Artigo } \\
\text { SERVILHA EAM, ARBACH } \\
\text { MP }\end{array}$ & 2011 & Transversal & $\begin{array}{l}84(28 \mathrm{em} \\
\text { tempo integral } \\
\text { e } 57 \text { horistas })\end{array}$ & $\begin{array}{l}33-64 \\
\text { anos } \mathrm{TI} \\
32-74 \\
\text { anos } \mathrm{H}\end{array}$ & $\begin{array}{c}\mathrm{TI} \\
71,4 \% \mathrm{~F} \\
28,6 \% \mathrm{M} \\
\mathrm{H} \\
75 \% \mathrm{~F} \\
25 \% \mathrm{M}\end{array}$ & $\begin{array}{l}\text { Carregar peso, indisciplina na sala, supervisão constante, local } \\
\text { de descanso inadequado e estresse no trabalho associam-se } \\
\text { com queixas vocais, osteoarticulares, emocionais e auditivas. }\end{array}$ \\
\hline $\begin{array}{l}\text { Artigo } \\
\text { CARAN VCS et al. }\end{array}$ & 2011 & Transversal & 54 & $\begin{array}{l}20-60 \\
\text { anos }\end{array}$ & $\begin{array}{l}92,6 \% \mathrm{~F} \\
7,4 \% \mathrm{M}\end{array}$ & $\begin{array}{l}\text { Riscos ocupacionais psicossociais presentes no contexto } \\
\text { acadêmico foram percebidos como influenciadores na saúde. }\end{array}$ \\
\hline $\begin{array}{l}\text { Tese de doutorado } \\
\text { ALVES LA }\end{array}$ & 2011 & Transversal & 17 & $31-47$ anos & $100 \% \mathrm{~F}$ & $\begin{array}{l}\text { O uso prolongado da voz não promoveu alterações } \\
\text { significativas quanto a intensidade vocal e extensão dinâmica. } \\
\text { A análise acústica apresentou elevação dos parâmetros, } \\
\text { frequência fundamental e proporção harmônica ruído. }\end{array}$ \\
\hline $\begin{array}{l}\text { Dissertação de mestrado } \\
\text { DALAGASPERINA P, } \\
\text { MONTEIRO JK }\end{array}$ & 2012 & $\begin{array}{l}\text { Metodologia } \\
\text { qualitativa }\end{array}$ & 9 & $31-52$ anos & $\begin{array}{l}66,6 \% \mathrm{~F} \\
33,3 \% \mathrm{M}\end{array}$ & $\begin{array}{l}\text { A sobrecarga de trabalho dentro e fora do ambiente acadêmico, } \\
\text { os prazos e as cobranças, as dificuldades de relacionamento } \\
\text { com a chefia e com os alunos e os prejuízos na saúde, } \\
\text { representam os principais fatores de estresse. }\end{array}$ \\
\hline $\begin{array}{l}\text { Artigo } \\
\text { TERRA FS, MARZIALE } \\
\text { MHP, ROBAZZI MLCC }\end{array}$ & 2012 & Transversal & $\begin{array}{l}71 \text { (39 } \\
\text { instituição } \\
\text { pública e } 32 \\
\text { instituição } \\
\text { privada) }\end{array}$ & $31-40$ anos & $\begin{array}{l}\text { Predomínio sexo } \\
\text { feminino }\end{array}$ & $\begin{array}{l}\text { Com relação à classificação da autoestima, baseada nos } \\
\text { pontos de corte, não houve diferença significativa entre os dois } \\
\text { grupos ( } p=0,170) \text {, sendo que o percentual de autoestima média } \\
\text { foi de } 17,9 \% \text { nos docentes da universidade pública e de } 34,4 \% \text {, } \\
\text { nos da universidade privada. A autoestima baixa ocorreu em } \\
\text { apenas um docente da universidade pública }(2,6 \%)\end{array}$ \\
\hline $\begin{array}{l}\text { Tese de doutorado } \\
\text { MIGUEL MEGB }\end{array}$ & 2012 & Transversal & $\begin{array}{l}90 \text { (60 } \\
\text { instituição } \\
\text { pública e } 30 \\
\text { instituição } \\
\text { privada) }\end{array}$ & $24-58$ anos & $\begin{array}{l}87,7 \% \mathrm{~F} \\
12,2 \% \mathrm{M}\end{array}$ & $\begin{array}{l}\text { Não foram identificadas condições de vulnerabilidade ao } \\
\text { estresse elevado. A qualidade de vida geral e as condições de } \\
\text { saúde foram consideradas boas }(64,4 \%) \text {, sendo mais bem } \\
\text { avaliados os domínios físico }(72,8 \%) \text { e psicológico ( } 69,35 \%) \text {. } \\
\text { Houve correlação positiva entre resiliência e qualidade de vida }\end{array}$ \\
\hline $\begin{array}{l}\text { Artigo } \\
\text { SANCHEZ HM et al. }\end{array}$ & 2013 & Transversal & 36 & $20-60$ anos & Não relatado & $\begin{array}{l}\text { A incidência de dores musculoesqueléticas nos docentes é alta, } \\
\text { independente de idade, altura, tempo e jornada de trabalho. }\end{array}$ \\
\hline $\begin{array}{l}\text { Dissertação de mestrado } \\
\text { ALBUQUERQUE AL }\end{array}$ & 2013 & Transversal & 143 & $22-75$ anos & $\begin{array}{l}\text { IES pública } \\
69,3 \% \mathrm{~F} \\
30,7 \% \mathrm{M} \\
\text { IES privada } \\
49,1 \% \mathrm{~F} \\
50,9 \% \mathrm{M}\end{array}$ & $\begin{array}{l}\text { Os docentes das IES públicas e privadas estão expostos a } \\
\text { fatores desconfortáveis para o desenvolvimento de suas } \\
\text { atividades, sendo o acúmulo de atividades uma característica } \\
\text { dos docentes de IES privadas. Exposição a poeira e produtos } \\
\text { químicos são mais comuns em IES públicas. Ambas } \\
\text { instituições possuem os prejudiciais hábitos de falar muito e } \\
\text { falar alto, o que implica no abuso vocal e predisposição à } \\
\text { disfonia. }\end{array}$ \\
\hline
\end{tabular}




\begin{tabular}{|c|c|c|c|c|c|c|}
\hline SOUSA AM & & & & mais & $55,7 \% \mathrm{M}$ & $\begin{array}{l}\text { afastamento, com destaque para os transtornos mentais e } \\
\text { comportamentais, e doenças do sistema musculoesquelético e } \\
\text { do tecido conjuntivo. }\end{array}$ \\
\hline $\begin{array}{l}\text { Artigo } \\
\text { LIMA MFEM. e LIMA FILHO } \\
\text { DO }\end{array}$ & 2009 & Transversal & 49 & $25-63$ anos & $\begin{array}{l}71,4 \% \mathrm{~F} \\
28,6 \% \mathrm{M}\end{array}$ & $\begin{array}{l}\text { É alta a prevalência de sintomas osteomusculares, assim como } \\
\text { a percepção da relação e intensificação com a atividade de } \\
\text { lecionar, sugerindo distúrbios osteomusculares relacionados ao } \\
\text { trabalho. }\end{array}$ \\
\hline $\begin{array}{c}\text { Artigo } \\
\text { COSTA LST et al. }\end{array}$ & 2013 & Transversal & 169 & $28-64$ anos & $\begin{array}{l}41,4 \% \mathrm{~F} \\
58,6 \% \mathrm{M}\end{array}$ & $\begin{array}{l}\text { A prevalência da Síndrome de Burnout encontrada é motivo de } \\
\text { preocupação e merece atenção, não só pelos danos que } \\
\text { provoca à saúde física, mental e social do profissional, mas } \\
\text { também pela influência na qualidade de ensino. }\end{array}$ \\
\hline $\begin{array}{c}\text { Dissertação de mestrado } \\
\text { RIBEIRO LH }\end{array}$ & 2015 & Transversal & 50 & Não relatado & $\begin{array}{c}40,7 \% \mathrm{~F} \\
50,4 \mathrm{M}\end{array}$ & $\begin{array}{l}\text { Os resultados da pesquisa quanto ao ambiente sonoro indicam } \\
\text { que as salas de aula apresentam problemas de excesso de } \\
\text { ruído e reverberação, com um alto risco para o } \\
\text { desenvolvimento de distúrbio de voz. }\end{array}$ \\
\hline $\begin{array}{c}\text { Dissertação de mestrado } \\
\text { COSTA DL }\end{array}$ & 2016 & $\begin{array}{l}\text { Metodologia } \\
\text { qualitativa }\end{array}$ & 7 & $28-69$ anos & $\begin{array}{l}85 \% \mathrm{~F} \\
15 \% \mathrm{M}\end{array}$ & $\begin{array}{l}\text { O absenteísmo está associado a um crescimento dos } \\
\text { transtornos mentais e comportamentais em comparação com } \\
\text { as outras causas de afastamentos. O ambiente de trabalho } \\
\text { predispõe a esgotamento profissional, alcoolismo, dependência } \\
\text { química, transtornos depressivos e ansiosos, assédio moral e } \\
\text { suicídio. } \\
\text { A prática do ensino em sala de aula e o reconhecimento } \\
\text { profissional são gratificantes. }\end{array}$ \\
\hline $\begin{array}{l}\text { Dissertação de mestrado } \\
\text { RIBEIRO AZCR }\end{array}$ & 2016 & Transversal & 494 & $\begin{array}{c}20-60 \text { anos ou } \\
\text { mais }\end{array}$ & $\begin{array}{l}47 \% \mathrm{~F} \\
53 \% \mathrm{M}\end{array}$ & $\begin{array}{l}\text { O professor universitário acumula atribuições e } \\
\text { responsabilidades que elevam os indices de morbidade e } \\
\text { absenteísmo, bem como altera sua prática docente. As } \\
\text { doenças que somaram mais dias de afastamentos foram as do } \\
\text { sistema osteomuscular com } 12,9 \% \text { e transtornos mentais e } \\
\text { comportamentais com } 12,6 \% \text {. }\end{array}$ \\
\hline $\begin{array}{c}\text { Artigo } \\
\text { LIMA Jr JPL, SILVA TFA }\end{array}$ & 2014 & Estudo transversal & 49 & $25-63$ anos & $71,4 \% \mathrm{~F} / 28,6 \% \mathrm{M}$ & $\begin{array}{l}\text { É alta a prevalência de sintomas osteomusculares, assim como } \\
\text { a percepção da relação e intensificação com a atividade de } \\
\text { lecionar, sugerindo distúrbios osteomusculares relacionados ao } \\
\text { trabalho. }\end{array}$ \\
\hline
\end{tabular}

